\title{
Aide à la validation des méthodes en Toxicologie et Suivi Thérapeutique Pharmacologique
}

\section{Help for validation of analytical procedures in Toxicology and Therapeutic Drug Monitoring}

Groupe de travail "Accréditation" de la Société Française de Toxicologie Analytique

Coordonnateur : Anne GRUSON, Laboratoire de Biochimie-Toxicologie, Centre Hospitalier, 57, avenue Winston Churchill - 62022 ARRAS Cedex - Tél : 0321211085 - E-mail : anne.gruson@ch-arras.fr

Membres du groupe de travail : Bernard DINGEON, Marie-Françoise DREYFUSS, Yvan GAIlLARD, Anne LE BOUIL, Michel LHERMITTE, Stéphanie MAZZEGA, Mustapha MOULSMA, Gilbert PÉPIN, Martine PERRIN, Jocelyne PLESSE, Olivier ROUSSEL, Marie-Thérèse THEVENOT, Élisabeth VINNER, Marc DEVEAUX (membre associé)

(Accepté pour publication le 31 octobre 2005)

\section{SOMMAIRE}

I. Introduction

II. État des lieux - Contexte réglementaire et normatif

III. Quelles sont les informations pertinentes à connaître ?

IV. Choix préalable à la vérification des critères de performance et limites d'acceptabilité de la méthode, des vérifications expérimentales, des vérifications bibliographiques

V. Vérification d'une méthode d'analyse quantitative - Contenu du dossier

V.1 Vérification initiale d'une méthode quantitative

V.2 Confirmation des performances en routine

VI. Vérification d'une méthode d'analyse qualitative - Contenu du dossier

VI. 1 Vérification initiale d'une méthode qualitative

VI. 2 Confirmation des performances en routine

VII. Vérification d'une méthode d'analyse assimilable au quantitatif à résultat qualitatif - contenu du dossier VII. 1 Vérification initiale d'une méthode assimilable au quantitatif

VII. 2 Confirmation des performances en routine

VIII. Maîtrise de la documentation - Méthodologie

IX. Exemples méthodologiques

IX.1 Fidélité

IX.2 Justesse (biais)

DX.3 Contamination

IX.4 Limite de détection et limite de quantification

IX.5 Linéarité

IX.6 Stabilité

IX.7 Corrélation de méthodes

X. Terminologie - Autres définitions

XI. Bibliographie 


\section{Introduction}

Ce document a pour but d'expliciter ce qui est entendu par les aspects de "validation de méthode" dans le cadre précis des laboratoires engagés ou souhaitant s'engager dans une démarche de qualité dans le domaine des analyses en Toxicologie et Suivi Thérapeutique Pharmacologique, et notamment dans une démarche d'accréditation COFRAC selon le programme 168. Dans ce domaine, la problématique de la "validation" repose sur le fait que les méthodes d'analyses ne sont pas issues de normes publiées. Cette "validation" s'inscrit alors dans une vérification des performances annoncées par le fabricant ou souhaitées par le laboratoire, lors de la mise en application des méthodes d'analyses dans son laboratoire. Ceci afin de lui apporter confirmation et preuve de la validité des résultats rendus (phase de "qualification"), par rapport à ses propres besoins. Cette validation est à prendre en terme de maîtrise du processus analytique, au niveau initial.

La validation d'une méthode devra s'envisager en fonction des différents contextes auxquels sera confronté le biologiste ou le responsable technique. "La validation est toujours un équilibre entre les coûts, les risques et les possibilités techniques" (extrait de 1'ISO/CEI 17025, § 5.4.5.3 note 3, et NF EN ISO 15189), et le temps. On entend par validation, "[...] la confirmation par examen et apport de preuves objectives du fait que les prescriptions particulières en vue d'une utilisation déterminée sont remplies." (extrait de l'ISO/CEI 17025, § 5.4.5.1).

Au préalable, le biologiste ou le responsable technique devra lire attentivement le dossier des fournisseurs (notices, fiches techniques, références bibliographiques, ...). Il devra évaluer et apprécier ces informations à la lueur de recommandations ou de textes réglementaires, des critères de performance proposés par des sociétés savantes, des attentes du prescripteur ou du requérant.

Dans un deuxième temps la validation sera effectuée. Le champ et la profondeur de cette évaluation dépendront des circonstances et de chaque cas particulier. Cette validation devra être initiale, puis se continuer dans le temps (changement de lots de réactifs [si effet de lot connu], extensions analytiques, maintenance lourde,...). Dans certains cas cette validation sera une confirmation des performances d'une méthode déjà en cours d'utilisation.

Les critères fondamentaux de l'évaluation de la méthode (exemple dossier de marquage CE) sont en principe déterminés par le fabricant. Si les coffrets réactifs et systèmes sont utilisés strictement dans les conditions préconisées par le fabricant, les méthodes qui y font appel sont prises en compte comme des méthodes normalisées. Dans ce cas, le laboratoire doit uniquement valider (vérifier) la mise en application dans son environnement propre par rapport à des critères et des limites acceptables (spécifications) qu'il s'est fixé, pour correspondre aux besoins de ses clients. Il n'est en effet pas demandé aux laboratoires d'effectuer de nouveau l'évaluation des méthodes ou des automates, études qui ont déjà été réalisées par le fournisseur ou le fabricant, qui eux ne connaissent pas ces besoins.

Note 1 : De la définition de la validation découle la méthodologie de la conduite d'un processus de validation. "Les prescriptions particulières" identifient les caractéristiques, par exemple les spécifications préalablement définies sur certains critères également choisis, conditions qui doivent être remplies pour déclarer la méthode valide.

Note 2 : Ce document a été élaboré à partir du guide de validation des méthodes en biologie médicale : « document LAB GTA 04 (juin 2004).

\section{II. État des lieux - Contexte réglementaire et normatif}

Ce guide sert à établir un protocole d'évaluation des méthodes utilisées dans un laboratoire. Cette évaluation permet de constituer le dossier de validation des méthodes utilisées dans le laboratoire.

Cette validation est une exigence de la norme NF EN ISO/CEI 17025 dans son paragraphe $\S 5.4$ traitant des "Méthodes d'essai et d'étalonnage et validation des méthodes", et plus précisément au $\S 5.4 .5$ Validation des méthodes, qui est définie au § 5.4.5.1 : "La validation est la confirmation par examen et l'apport de preuves objectives du fait que les prescriptions particulières en vue d'une utilisation prévue déterminée sont remplies."

Le paragraphe 5.4.2 indique que: "Lorsque le client ne spécifie pas la méthode à utiliser, le laboratoire doit sélectionner des méthodes appropriées qui ont été publiées dans des normes internationales, régionales ou nationales, 
par des organisations techniques de renom ou dans des textes ou revues scientifiques spécialisés, ou spécifiées par le fabricant de l'équipement. Des méthodes développées par le laboratoire ou des méthodes adoptées par le laboratoire peuvent également être employées si elles conviennent à l'usage prévu et qu'elles ont été validées. Le client doit être informé de la méthode choisie. Le laboratoire doit confirmer qu'il peut correctement appliquer des méthodes normalisées avant de les mettre en auvre pour des essais ou des étalonnages. En cas de changement de la méthode normalisée, la confirmation doit être répétée."

Cette exigence figure dans le Guide de Bonnes Exécutions des Analyses de Biologie Médicale (Arrêté du 26 novembre1999 relatif à la bonne exécution des analyses de biologie médicales) : Définition des termes Evaluation (§ 2.6), Qualification ( $(2.11)$, Transférabilité ( $\$ 2.13)$, Valeurs de référence ( $(2.14)$, Validation $(\S 2.15)$; dans le $\S$ $1.1 \mathrm{c}$ concernant les installations, l'équipement, l'instrumentation, les produits fongibles et les réactifs ".... s'assurer que les installations, l'équipement et l'instrumentation du laboratoire sont fonctionnels."

Dans la nouvelle norme ISO 15189 , la validation est retrouvée au niveau du $\S 5.3 .2, \S 5.3 .4$ et son point. h) mais surtout dans le paragraphe 5.5.1: "Le laboratoire doit utiliser des procédures analytiques, incluant celle de sélection et d'aliquotage d'échantillons, qui correspondent aux besoins des utilisateurs des prestations de laboratoire et qui conviennent à chaque type d'analyse. Les procédures analytiques conseillées sont celles qui ont été publiées dans des manuels bien établis et faisant autorité, dans des textes et des journaux revus par des experts ou des recommandations régionales, nationales ou internationales. Si des procédures internes sont utilisées, elles doivent être validées de manière appropriée pour l'utilisation prévue et parfaitement documentées.", et le paragraphe 5.5.2 : "Le laboratoire doit utiliser uniquement des procédures validées pour s'assurer qu'elles conviennent à l'utilisation prévue. Les validations doivent être aussi approfondies que nécessaires pour répondre aux besoins de l'application ou du domaine d'application concerné(e). Le laboratoire doit enregistrer les résultats obtenus et la procédure utilisée pour la validation.

Les méthodes et les procédures sélectionnées doivent être évaluées et donner des résultats satisfaisants avant d'être utilisées pour les analyses médicales. Une revue des procédures par le directeur du laboratoire ou une personne désignée doit être réalisée à l'origine à intervalles définis. Ces revues sont généralement effectuées une fois par an. Ces revues doivent être documentées." Le paragraphe suivant (\$ 5.5.3) développe cet aspect de validation en précisant "[...] Les performances et l'aptitude à l'emploi prévu de chaque nouvelle version des trousses de réactifs prêts à l'emploi présentant des modifications importantes en termes de réactifs ou de procédure doivent être vérifiées. Tout changement de la procédure doit être daté et faire l'objet d'une autorisation comme pour les autres procédures."

Le paragraphe 5.3.2 quant à lui indique que "Il doit être démontré (lors de l'installation et au cours de l'utilisation courante) que le matériel est capable d'atteindre les performances requises et qu'il est conforme aux spécifications se rapportant aux analyses.

La direction du laboratoire doit élaborer un programme de surveillance régulière permettant de démontrer l'adéquation de l'étalonnage et du fonctionnement des instruments, des réactifs, et des systèmes analytiques. Elle doit également instaurer un programme de maintenance préventive documenté et enregistré [...] respectant, au minimum, les recommandations du fabricant."

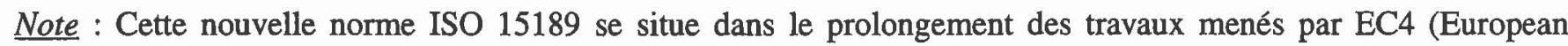
Communities Confederation of Clinical Chemistry) publiés dans l'article : Essential Criteria for Quality Systems of Medical Laboratories (Eur J Clin Chem Clin Biochem 1997 ; 35(2) : 123-132 @ 1997 by Walter de Gruyter Berlin New York). Cet article est consultable à partir du site : http://www.uni-oldenburg.de/ec4/ec4qual.htm\#ECText.

Outre la compétence du biologiste ou du responsable technique, l'utilisation et la gestion correcte des Contrôles de Qualité (CQI, CQE), la validation permet d'assurer la qualité de la prestation d'analyse et du résultat. Cette validation sera mise à profit pour estimer les incertitudes des mesures du laboratoire, notion présente dans le GBEA : " $\$$ 4. Expression des résultats et comptes rendus d'analyses, 4.1. Expression des résultats : L'expression des résultats doit être précise et sans équivoque. Les valeurs de référence doivent être indiquées. La méthode d'analyse et/ou les réactifs utilisé(e)(s) doivent être mentionné(e)(s) chaque fois qu'ils peuvent influer sur l'expression du résultat ainsi que lorsque la réglementation l'exige. Pour les résultats quantitatifs, le cas échéant, les performances analytiques de la méthode peuvent être indiquées. Les unités du système international (SI) doivent être utilisées quand elles existent." 
Cette estimation de l'incertitude de mesure se retrouve dans la norme ISO/CEI 17025 au § 5.4.6 "Estimation de l'incertitude de mesure", avec au § 5.4.6.2 "Les laboratoires d'essais doivent aussi posséder et appliquer des procédures pour estimer l'incertitude de mesure. Dans certains cas, la nature de la méthode d'essai exclut un calcul rigoureux, métrologiquement et statistiquement valable, de l'incertitude de mesure. Dans de tels cas, le laboratoire doit au moins tenter d'identifier toutes les composantes de l'incertitude et faire une estimation raisonnable, tout en assurant que la manière d'en rendre compte ne donne pas une impression erronée de l'incertitude. Une estimation raisonnable doit se baser sur une connaissance de la performance de la méthode et sur le domaine de la mesure et faire appel, par exemple, à l'expérience acquise et aux données de validation antérieures."

Cette approche des incertitudes est aussi une exigence de la nouvelle norme ISO 15189. Dans le paragraphe traitant de la validation, $\$ 5.5 .3$, son point $\mathrm{h}$ ) indique "les sources potentielles de variation des résultats." En outre, dans le §5.6.2, il est indiqué : "Le laboratoire doit déterminer l'incertitude des résultats, dans le cas où cela est pertinent et possible. Toutes les composantes importantes de l'incertitude doivent être prises en compte. Les sources contribuant à l'incertitude peuvent inclure l'échantillonnage, la préparation des échantillons, la sélection des aliquotes d'échantillon, les calibrateurs, les matériaux de référence, les grandeurs d'entrée, l'équipement utilisé, les conditions expérimentales, l'état de l'échantillon et les changements de manipulateurs."

Le biologiste, ou le responsable technique, doit pouvoir mettre à disposition du demandeur d'analyses, en cas de besoin, une approche de l'évaluation des incertitudes de mesure.

\section{Quelles sont les informations pertinentes à connaître ?}

D'une manière générale, une méthode d'analyse présente plusieurs caractéristiques essentielles que le laboratoire se doit de connaître. La connaissance de ces paramètres peut être acquise par la bibliographie et/ou par l'expérimentation sur site.

Schématiquement, on distinguera trois types de méthodes d'analyse que 1'on sera amené à traiter différemment :

\section{Les analyses de type quantitatif vrai :}

Elles fournissent un résultat chiffré, sur une échelle continue dont les limites basses et hautes sont connues, en relation directe avec une quantité ou une activité donnée de l'analyte à mesurer.

Ex : Dosage de médicaments dans le cadre du Suivi Thérapeutique Pharmacologique et dosage d'autres xénobiotiques en Toxicologie. Analyses réalisées par méthodes immunologiques et/ou chromatographiques.

\section{Les analyses de type qualitatif :}

Le résultat de ce type d'analyse n'apporte pas d'information sur la quantité de l'analyte, mais seulement sur sa présence ou sa non détection (positif ou non détecté).

Ex : Dépistage de xénobiotiques par méthodes chromatographiques. Les pics des chromatogrammes obtenus sont identifiés par les temps de rétention et par les spectres UV et/ou de masse. Ils peuvent être comparés à une bibliothèque prédéfinie de spectres.

\section{Les analyses assimilables à des analyses quantitatives :}

Dans ce cadre seront regroupées des analyses fournissant un résultat de type qualitatif, extrapolé à partir de la mesure d'une donnée quantifiable (Absorbance par exemple) avec un effet de seuil.

Ex : En Toxicologie, détermination d'une classe de molécules médicamenteuses (benzodiazépines, antidépresseurs tricycliques...), ou de substances illicites (amphétamines, opiacés, cannabinoïdes, cocaïniques...) avec des seuils prédéfinis. En Suivi Thérapeutique Pharmacologique (observance du traitement), mesure d'une molécule ciblée (méthadone, buprénorphine....). Les analyses sont réalisées selon le principe d'immunocompétition (EMIT, ELISA, CEDIA...). 
Selon l'appartenance de la méthode à un de ces trois types d'analyse ainsi définis, les informations pertinentes à connaître sont exposées dans le tableau ci-dessous :

\begin{tabular}{|l|l|l|}
\hline \multicolumn{1}{|c|}{ Méthode quantitative } & \multicolumn{1}{|c|}{ Méthode qualitative } & \multicolumn{1}{c|}{ Méthode assimilable au quantitatif } \\
\hline Spécificité & Spécificité & Spécificité \\
\hline Fidélité & NA & Fidélité \\
\hline Justesse (approche de la) & NA & NA \\
\hline Domaine d'analyse & NA & NA \\
\hline $\begin{array}{l}\text { Limite de détection } \\
\text { Limite de quantification }\end{array}$ & Limite de détection & Limite de détection \\
\hline Linéarité & NA & NA \\
\hline $\begin{array}{l}\text { Contamination entre échantillons } \\
\text { (s'il y a lieu) }\end{array}$ & $\begin{array}{l}\text { Contamination entre échantillons } \\
\text { (s'il y a lieu) }\end{array}$ & $\begin{array}{l}\text { Contamination entre échantillons } \\
\text { (s'il y a lieu) }\end{array}$ \\
\hline Stabilité & Stabilité & Stabilité \\
\hline Robustesse & Robustesse & Robustesse \\
\hline $\begin{array}{l}\text { Valeurs de référence } \\
\text { "ex-valeurs normales" }\end{array}$ & NA & NA \\
\hline Interférences & Interférences & Interférences \\
\hline $\begin{array}{l}\text { Corrélation avec méthode de référence ** } \\
\text { Corrélation avec méthode déjà } \\
\text { utilisée au laboratoire ** }\end{array}$ & $\begin{array}{l}\text { Corrélation avec méthode de référence ** } \\
\text { Coorrélation avec méthode déjà } \\
\text { utilisée au laboratoire ** }\end{array}$ & $\begin{array}{l}\text { Corrélation avec méthode de référence ** } \\
\text { Coorrélation avec méthode déjà } \\
\text { utilisée au laboratoire ** }\end{array}$ \\
\hline
\end{tabular}

(* NA : Non applicable) ** Chaque fois que cela est nécessaire

$\underline{\text { Note }}$ : Les paramètres de fidélité (répétabilité, fidélité intermédiaire et/ou de reproductibilité) d'une part, et de justesse d'autre part, constituent les composantes de l'incertitude de mesure, dans le calcul d'incertitude globale selon l'approche ISO 5725, en tant que composantes de type A. Associée aux composantes d'étalonnage le cas échéant et aux composantes de type B, l'incertitude globale peut alors être déterminée (voir chapitre IX. Exemples méthodologiques).

\section{Choix préalable à la vérification des critères de performance et limites d'acceptabilité de la méthode, des vérifications expérimentales, des vérifications bibliographiques}

A ce stade, il est fondamental de souligner que :

- Le choix des critères de performance (paramètres) et limites d'acceptabilité (spécifications, seuils) associés d'une méthode doit se faire préalablement à l'étude de validation.

- Ce choix est du ressort du biologiste ou du responsable technique.

- Ce choix doit refléter l'état de l'art et la pertinence toxicologique et clinique. Il pourra s'appuyer sur des recommandations de sociétés savantes ou de groupes de travail, ou issues de conférences de consensus, sur des publications scientifiques, sur des résultats de campagne de comparaisons interlaboratoires, etc...

- Les limites d'acceptabilité ainsi choisies doivent être adaptées et notifiées pour chacun des niveaux, pour chacun des analytes et pour chacune des matrices qui seront contrôlés dans l'étude.

Ces limites d'acceptabilité peuvent reposer sur différentes approches : l'intervalle des valeurs de référence (prise en compte de la variation biologique inter individuelle et de la variation analytique), les variations inter-individuelles et intra-individuelles, l'opinion des cliniciens et/ou des médecins légistes et l'état de l'art. 


\section{Vérification d'une méthode d'analyse quantitative - Contenu du dossier}

La vérification d'une méthode comprend une phase initiale, avant sa mise en œuvre effective en routine, et une phase de vérification continue et de confirmation des performances dans le cadre du fonctionnement normal et quotidien du laboratoire.

\section{V.1 Vérification initiale d'une méthode quantitative}

Dans ce cadre, le dossier devra apporter les éléments de vérification suivants, par recherche bibliographique et/ou par vérification sur site :

\begin{tabular}{|l|l|l|}
\hline \multicolumn{1}{|c|}{ PARAMEÉTRES A VÉRIFIER ET/OU CONNAITTRE } & \multicolumn{1}{c|}{ Bibliógraphification sur site } \\
\hline Spécificité & Oui & Non \\
\hline Fidélité & Oui & Oui \\
\hline Justesse (approche de la) & Oui & Si possible \\
\hline Domaine d'analyse & Oui & Si besoin \\
\hline $\begin{array}{l}\text { Limite de détection } \\
\text { Limite de quantification }\end{array}$ & Oui & Si besoin \\
\hline Linéarité & Oui & Si besoin \\
\hline Contamination entre échantillons (s'il y a lieu) & Oui & $\begin{array}{l}\text { Oui pour les paramètres } \\
\text { sensibles }\end{array}$ \\
\hline Stabilité & Oui & Si besoin \\
\hline Robustesse & Oui & Non \\
\hline Interférences & Oui & Si besoin \\
\hline Valeurs de référence "ex-valeurs normales" & Oui & Non \\
\hline $\begin{array}{l}\text { Corrélation avec méthode de référence } \\
\text { Corrélation avec méthode déjà utilisée au laboratoire }\end{array}$ & $\begin{array}{l}\text { Oui } \\
\text { Si existe }\end{array}$ & $\begin{array}{l}\text { Non } \\
\text { Si besoin }\end{array}$ \\
\hline Le dossier doit conclure sur l'avis d'aptitude ou d'inaptitude de la méthode ou du système analytique. \\
\hline
\end{tabular}

Certains de ces éléments peuvent éventuellement être vérifiés au début de la mise en œuvre en routine de la méthode (la fidélité intermédiaire notamment).

Le dossier de vérification peut renvoyer à d'autres documents (bibliographie, notices fournisseurs, documents internes au laboratoire, etc.), correctement identifiés et accessibles.

\section{V.2 Confirmation des performances en routine}

Pour maîtriser pleinement une méthode, l'utilisation, la gestion et le suivi de contrôles de qualité internes (CQI) et externes (CQE) si disponibles, sont indispensables.

Leur exploitation statistique permet de vérifier et de confirmer les éléments suivants :

- Fidélité,

- Justesse, ou du moins en faire une approche en l'absence de matériau de référence,

- Vérification (et adaptation éventuelle) des valeurs de références de la méthode pour la population du laboratoire.

\section{Vérification d'une méthode d'analyse qualitative - Contenu du dossier}

\section{VI.1 Vérification initiale d'une méthode qualitative}

Il sera souvent très difficile pour les laboratoires d'obtenir des échantillons positifs (ou dépourvus d'analyte) en nombre suffisant pour vérifier la spécificité et la limite de détection d'une méthode. En raison d'un recrutement forcément insuffisant, cela ne sera possible que dans les laboratoires importants. 
La vérification bibliographique critique prendra donc ici toute son importance. Inversement, la vérification expérimentale sur site sera plus réduite, et s'appuiera fortement sur l'étude des performances des Contrôles de Qualité Externes (CQE) si disponibles.

Dans ce cadre, le dossier pourra apporter les éléments de vérifications suivants :

\begin{tabular}{|l|l|l|}
\hline \multicolumn{1}{|c|}{ PARAMÈTRES A VÉRIFIER ET/OU CONNAITRE } & \multicolumn{1}{|c|}{ Bibliographie } & Vérification sur site \\
\hline Spécificité & Oui & Non \\
\hline Limite de détection & Oui & Non \\
\hline Contamination entre échantillons (s'il y a lieu) & Oui & Oui \\
\hline Stabilité & Oui & Si besoin \\
\hline Robustesse & Oui & Non \\
\hline Corrélation avec méthode de référence & Oui & Non \\
Corrélation avec méthode déjà utilisée au laboratoire & Si existe & Si besoin \\
\hline Le dossier doit conclure sur l'avis d'aptitude ou d'inaptitude de la méthode ou du système analytique. \\
\hline
\end{tabular}

Le dossier de vérification peut renvoyer à d'autres documents (bibliographie, notices foumisseurs, documents internes au laboratoire, etc.), correctement identifiés et accessibles.

Note à propos de l'étude de contamination :

* Cette étude de contamination inter-échantillons est à mener pour tous les systèmes analytiques.

* Cette étude sera à répéter au moindre doute quant au fonctionnement correct du système analytique, et en particulier du système de lavage et/ou de décontamination.

\section{VI.2 Confirmation des performances en routine}

Pour maîtriser pleinement une méthode, là aussi, l'utilisation, la gestion et le suivi de contrôles de qualité intemes (CQI) et externes (CQE) si disponibles sont indispensables.

La surveillance de la spécificité et de la limite de détection passe par l'étude des CQI et CQE, si disponibles (performances du laboratoire, comparaison avec les autres méthodes).

\section{Vérification d'une méthode d'analyse assimilable au quantitatif à resultat qualitatif - Contenu du dossier}

Ce cas de figure est très proche de celui des méthodes purement qualitatives, mais certaines caractéristiques supplémentaires peuvent être vérifiées sur site.

\section{VII.1 Vérification initiale d'une méthode assimilable au quantitatif}

S'agissant de méthodes fournissant un résultat final de type qualitatif, les laboratoires se heurteront à la même difficulté pour obtenir des échantillons positifs (ou dépourvus d'analyte) en nombre suffisant pour vérifier la spécificité et la limite de détection.

La vérification bibliographique critique prendra donc ici toute son importance. Le fabricant devra fournir ces éléments en s'appuyant sur des études menées par des experts indépendants. Inversement, la vérification expérimentale sur site sera plus réduite que pour une méthode quantitative, mais elle permettra néanmoins de vérifier certains éléments. 
Dans ce cadre, le dossier pourra apporter les éléments de vérifications suivants :

\begin{tabular}{|c|c|c|}
\hline PARAML̀TRES A VÉRIFIER ET/OU CONNAITRE & Bibliographie & Vérification sur site \\
\hline Spécificité & Oui & Non \\
\hline Fidélité & Oui & Oui \\
\hline Limite de détection & Oui & NA \\
\hline Contamination entre échantillons (s'il y a lieu) & Oui & Oui \\
\hline Stabilité & Oui & Si besoin \\
\hline Robustesse & Oui & Non \\
\hline Interférences & Oui & NA \\
\hline Corrélation avec méthode de référence & Oui & Non \\
\hline Corrélation avec méthode déjà utilisée au laboratoire & Si existe & Si besoin \\
\hline
\end{tabular}

(*NA : Non Applicable)

Le dossier de vérification peut renvoyer à d'autres documents (bibliographie, notices fournisseurs, documents internes au laboratoire, etc.), correctement identifiés et accessibles.

Note à propos de l'étude de contamination :

* Cette étude de contamination inter-échantillons est à mener pour tous les systèmes analytiques.

* Cette étude sera à répéter au moindre doute quant au fonctionnement correct du système analytique, et en particulier du système de lavage et/ou de décontamination.

\section{VII.2 Confirmation des performances en routine}

La surveillance de la spécificité et de la limite de détection passe :

* Par la comparaison avec les résultats obtenus par d'autres méthodes complémentaires ou par des méthodes de référence mises en œuvre pour vérifier un premier résultat,

* Par l'étude des CQE, si disponibles (performances du laboratoire, comparaison avec les autres méthodes).

La fidélité devra être également régulièrement vérifiée, surtout pour les valeurs proches du seuil décisionnel ("cut-off").

\section{Maîtrise de la documentation - Méthodologie}

Cette partie du guide est importante car si beaucoup d'informations et résultats sont collectés, ceux-ci doivent faire l'objet d'un document cohérent et clair, avec une acceptation formelle par le laboratoire de la validité opérationnelle de ses techniques, c'est-à-dire une décision ou déclaration quant à la validation de la méthode. Le laboratoire pourra présenter ses dossiers suivant le schéma suivant, qui pourra être celui de sa procédure (cf. document COFRAC $\mathrm{N}^{\circ} 1133$ ) ou de sa méthodologie :

* Présentation de la méthode, de l'appareillage et du mode opératoire. But de la vérification/validation. Domaine d'application,

* Détermination des critères de performances (paramètres) à vérifier (cf. chapitres IV, V, VI et VII),

* Détermination des spécifications ou limites acceptables (objectifs à atteindre) de ces critères à remplir (cf. chapitre IV),

* Vérification bibliographique,

* Mise en œuvre - Expérimentations (cf. chapitre VIII),

* Compilation et traitement statistique des données obtenues,

* Conclusion et décision quant à la validation opérationnelle de la méthode, au regard des spécifications (limites acceptables) initialement fixées. 


\section{Exemples méthodologiques}

Ce paragraphe décrit des principes méthodologiques ou des recommandations permettant de vérifier les paramètres essentiels d'une méthode :

* Fidélité,

* Justesse,

* Contamination inter-échantillons,

* Limite de détection, limite de quantification,

* Linéarité,

* Stabilité,

* Corrélation de méthodes,

* Vérification des valeurs de référence, ...

Les laboratoires pourront s'en inspirer. D'autres démarches pourront être possibles, elles devront être justifiées.

Remarque : Ce dossier peut dans certains cas être constitué au fil du travail du laboratoire. Dans ce cas la période doit être la plus courte possible, le rappel éventuel de résultats de patient devra être documenté. Il est souhaitable de prévoir une sérothèque particulière à ces essais afin de pouvoir assurer un raccordement entre deux méthodes successives. En cas de modification de méthode ou d'un couple analyseur/méthode il est souhaitable, dans la mesure du possible, de pouvoir disposer simultanément de la nouvelle et de l'ancienne méthode pour pouvoir mener des essais en parallèle.

Note : Dans le cadre de l'accréditation, le laboratoire doit présenter au COFRAC, deux éléments différents :

1. la procédure qui décrit les lignes directrices et les modalités du processus qu'il suit pour la validation des méthodes en général (cf. ce guide),

2. le dossier de validation de la méthode proprement dit, qui est un enregistrement (élément de preuve ; bibliographie, données brutes, calculs, conclusions du biologiste ou responsable technique).

\section{IX.1- Fidélité}

Les essais de répétabilité et de fidélité intermédiaire sont obligatoires.

\section{IX.1.1 - Répétabilité}

L'essai de répétabilité consiste à effectuer l'analyse d'un même échantillon pour le même analyte dans des conditions identiques : même opérateur, même lots de réactifs, même instrument, même calibrateur.

En pratique, cet essai sera réalisé au cours d'une même série. Il est recommandé d'utiliser au moins deux niveaux de concentration. Les niveaux seront choisis en fonction des valeurs physiopathologiques et/ou de la réglementation. Le nombre de déterminations dépendra du système analytique, du coût des réactifs, ... Cet effectif devra être $\geq 6$. La valeur statistique des résultats obtenus dépend de ces effectifs.

L'exploitation des résultats consiste à calculer la moyenne (m), l'écart-type (s) et le coefficient de variation (CV) des valeurs expérimentales de chaque série.

Le CV représentera la répétabilité de la méthode en \%. Il sera comparé au CV limite d'acceptabilité.

Le calcul sera répété pour chacune des matrices (sérum, urine, et sang total en médicolégal,...) soumises à analyse. Pour un même analyseur, ce calcul devra être effectué pour chaque analyte à mesurer.

\section{IX.1.2 - Fidélité intermédiaire}

L'essai de fidélité intermédiaire consiste à effectuer l'analyse d'un même échantillon pour le même analyte dans des conditions différentes : l'opérateur, les réactifs, l'appareillage, les calibrages et les gammes d'étalonnage, les jours peuvent être des données variables.

Il est recommandé d'utiliser au moins deux niveaux de concentration. Les niveaux seront choisis en fonction des valeurs physiopathologiques et/ou de la réglementation. 6 déterminations minimum sont nécessaires pour chacun des échantillons de validation.

Les modalités de calcul sont identiques à celles de la répétabilité, avec calcul de la moyenne (m), de l'écart-type (s) 
et du coefficient de variation (CV) sur les valeurs expérimentales de chaque série ; le CV ainsi calculé sera comparé au CV limite d'acceptabilité.

Le CV représentera la fidélité intermédiaire de la méthode, exprimé en \%.

Ce calcul sera répété pour chacune des matrices (sérum, urine, et sang total en médicolégal,...) soumises à analyse. Pour un même analyseur, ce calcul devra être effectué pour chaque analyte à mesurer.

Note : Pour des raisons pratiques, cet essai pourra éventuellement être mené après l'installation du système analytique et la mise en œuvre de la méthode, si et seulement si, les autres spécifications sont bien atteintes.

\section{IX.1.3- Reproductibilité}

L'essai de reproductibilité consiste à effectuer l'analyse d'un même échantillon dans différents laboratoires.

\section{2 - Justesse (biais)}

En Toxicologie et Suivi Thérapeutique Pharmacologique, il n'existe pas, à de rares exceptions près, d'échantillons de contrôles raccordés sur le plan métrologique à des étalons internationaux ; il est donc impossible de parler stricto sensu de "valeur vraie" et par là-même de justesse.

En l'absence de matériau de référence certifié, on pourra utiliser des échantillons utilisés pour des CQE réguliers et dont la commutabilité aura été vérifiée ou prouvée. Il est souhaitable (mais pas obligatoire) d'utiliser des échantillons de différentes origines commerciales.

La justesse (biais) sera estimée en comparant la moyenne obtenue $(\mathrm{m})$ lors de l'étude de répétabilité et/ou de fidélité intermédiaire à la valeur cible attendue assimilée à la valeur "vraie" (v) de l'échantillon testé.

Elle sera exprimée en pourcentage de la valeur cible, selon le calcul suivant :

$$
\text { Justesse en } \left.\%=\frac{(m-v}{v}\right) \times 100
$$

Cette justesse pourra aussi être contrôlée à l'aide des valeurs de référence qui pourront être comparées à celles de la littérature.

\section{Exemples d'application - Approche de l'inexactitude et calcul d'incertitude.}

Exemple 1 - Les paramètres de fidélité d'une part, et de justesse d'autre part, constituent les composantes de l'incertitude de mesure, dans le calcul d'incertitude globale selon l'approche ISO 5725, en tant que composantes de type A. Pour ce calcul, on prendra le cas de composantes indépendantes.

Ainsi, si $\mathrm{u}_{1}$ est l'incertitude de fidélité, celle-ci est égale à l'écart-type de fidélité intermédiaire :

$$
\mathrm{u}_{1}=\mathrm{s}_{\mathrm{Fid}}
$$

Pour ce qui est de l'incertitude de justesse, $\mathrm{u}_{2}$, elle est donnée par la relation :

$$
\mathrm{u}_{2}=\frac{\text { justesse }}{\sqrt{3}} \quad \text { (Loi rectangle) }
$$

En l'absence de matériau de référence, c'est-à-dire "d'étalon", de valeur "vraie" connue (avec une incertitude associée, $u_{\mathrm{ref}}$ ), le laboratoire peut utiliser pour calculer l'incertitude de justesse, les données de Contrôle de Qualité Externe (CQE), c'est-à-dire le biais moyen, l'écart-type de cette moyenne et toujours l'incertitude de la valeur cible, la valeur "vraie". $u_{2}$ peut alors s'exprimer différemment en fonction de ces différentes composantes.

Ces composantes d'incertitude, combinées à celle de matériau de référence $\left(\mathrm{u}_{\mathrm{ref}}\right)$, celles d'étalonnage $\left(\mathrm{u}_{\mathrm{et}}\right)$, et enfin aux composantes de type $\mathrm{B}\left(\mathrm{u}_{\mathrm{B}}\right)$, le cas échéant, permettent de déterminer l'incertitude globale, c'est-à-dire l'incertitude combinée $\left(\mathrm{u}_{\mathrm{c}}\right)$, puis l'incertitude élargie $\mathrm{U}$, selon la relation issue de la somme quadratique des composantes d'incertitude,

$$
\mathrm{u}_{\mathrm{c}}=\sqrt{\mathrm{u}_{1}^{2}+\mathrm{u}_{2}^{2}+\mathrm{u}_{\mathrm{ref}}^{2}+\mathrm{u}_{\mathrm{et}}^{2}+\Sigma \mathrm{u}_{\mathrm{B}}^{2}}
$$


Enfin, l'incertitude élargie U est alors,

$$
\mathrm{U}=2 . \mathrm{U}_{\mathrm{c}} \quad \text { (avec } « 2 »=\text { intervalle de confiance de } 95 \% \text {, dans le cas d'une distribution gaussienne). }
$$

Le résultat de la mesure analytique, $\mathrm{R}$, s'exprimera alors :

$$
\mathrm{R}=\text { valeur mesurée } \pm \mathrm{U} \text { (unité) }
$$

Exemple 2 - Une autre approche de l'incertitude peut être proposée (session d'étude SFSTP du 23/10/2002 ; Méthodes chromatographiques de dosage dans les milieux biologiques : stratégie de validation. STP Pharma pratiques 1997). Les études de répétabilité et de fidélité intermédiaire donnent accès à $S_{\mathrm{r}}^{2}$, variance de répétabilité et $\mathrm{S}_{\mathrm{g}}^{2}$ variance de fidélité intergroupes et $\mathrm{S}_{\mathrm{R}}^{2}$ variance de fidélité intermédiaire, telles que :

$$
\mathrm{S}_{\mathrm{R}}^{2}=\mathrm{S}_{\mathrm{r}}^{2}+\mathrm{S}_{\mathrm{g}}^{2}
$$

L'incertitude de mesure peut être alors exprimée ainsi :

$$
I C_{R}=\text { résultat } \pm t^{(\alpha, v)} \bullet \sqrt{\frac{S_{g}^{2}}{k}+\frac{S_{r}^{2}}{k \bullet n}}
$$

Avec $\alpha$ risque choisi, $v$ degrés de liberté de $\mathrm{S}_{\mathrm{R}}^{2}, k$ nombre de séries nécessaires à la détermination du résultat (condition de fidélité intermédiaire) et $\mathbf{n}$ nombre de répétitions nécessaires à la détermination du résultat (conditions de répétabilité).

Si $\mathrm{k}=\mathrm{n}=1$, l'incertitude peut s'exprimer comme :

$$
I C_{R}=\text { résultat } \pm t^{(\alpha, v)} \cdot \sqrt{S_{R}^{2}}
$$

Ainsi exprimée, l'incertitude devient un véritable outil pour l'analyste. En effet, lors de l'application en routine de la méthode validée, le nombre de série $\mathrm{k}$ et le nombre de répétition $\mathrm{n}$ peuvent être adaptés à une précision requise. Ex :

$$
I C_{R}=\text { résultat } \pm 2,10 \cdot \sqrt{\frac{4,56}{k}+\frac{11,27}{k \bullet n}}
$$

avec $\mathrm{k}=\mathrm{n}=1, \quad \mathrm{IC}_{\mathrm{R}}=$ résultat $\pm 8.35 \%$

avec $\mathrm{k}=1$ et $\mathrm{n}=2, \quad \mathrm{IC}_{\mathrm{R}}=$ résultat $\pm 6.70 \%$

avec $\mathrm{k}=2$ et $\mathrm{n}=1, \mathrm{IC}_{\mathrm{R}}=$ résultat $\pm 5.90 \%$

aveć $\mathrm{k}=2$ et $\mathrm{n}=2, \quad \mathrm{IC}_{\mathrm{R}}=$ résultat $\pm 4.74 \%$, etc...

\section{3 - Contamination}

Une étude des contaminations inter-échantillons sera effectuée de la façon suivante :

Après rinçage de l'appareil, un échantillon élevé (ou positif fort) sera analysé 3 fois consécutivement (H1, H2, H3 de moyenne $\overline{\mathrm{H}}$ ) suivi d'un échantillon bas également passé 3 fois (B1, B2, B3)

Le pourcentage de contamination entre les échantillons peut être calculé selon la formule suivante :

$$
\text { Contamination en } \%=\frac{(B 1-B 3)}{\bar{H}} \times 100
$$

Note : Compte tentu de l'importance clinique de certains paramètres, le niveau de la contamination doit être maintenue à zéro ou entraîner des règles de réanalyse argumentées. 


\section{4 - Limite de détection et limite de quantification}

\section{IX.4.1- Limite de détection}

La limite de détection est le plus petit signal exprimé en quantité ou en concentration qui peut être distingué avec une probabilité donnée d'un blanc réalisé dans les meilleures conditions. C'est la quantité minimale détectable pour laquelle la réponse (en signal mesuré) peut être distinguée avec une probabilité donnée d'un blanc.

L'étude de la limite de détection est basée sur l'analyse statistique de la différence de signaux observés pour les blancs et les échantillons.

Méthode 1 : Pour l'estimer, on pourra effectuer 30 mesures répétées des blancs (matrice dépourvue de la substance à doser), dans une même série, et on calculera l'écart-type (s), exprimé en concentration, de ces 30 mesures.

La limite de détection peut être calculée selon la formule suivante :

Limite de détection $=$ moyenne +3 écarts-type

Méthode 2 : Une limite de détection peut être déterminée par excès et correspondre à la limite de quantification.

\section{IX.4.2- Limite de quantification}

La limite de quantification correspond à la plus petite valeur exprimée en concentration, rendue avec une confiance acceptable. C'est la valeur pour laquelle le CV et l'écart à la valeur théorique sont inférieurs à la limite d'acceptabilité.

Méthode 1 : la limite de quantification peut être calculée à partir des mesures de blanc

Limite de quantification $=$ moyenne +10 écarts-type

Méthode 2 : Il est possible de réaliser des dilutions du calibrateur ou de l'échantillon de Contrôle de Qualité Interne le plus bas (différent de 0 ) avec le blanc échantillon, jusqu'à satisfaire la limite d'acceptabilité dans les mêmes conditions que les études de fidélité ( $\mathrm{n} \geq 6$ )

Pour déterminer la limite de quantification, on calculera, pour chaque série de mesures des différentes dilutions, l'écart-type (s), le Coefficient de Variation (CV) et l'écart de la moyenne (m) à la valeur théorique :

Limite de quantification = valeur pour laquelle le CV et l'écart à la valeur théorique sont inférieurs à la limite d'acceptabilité

\section{5 - Linéarité}

A partir d'une solution mère d'analytes, des solutions de concentrations différentes seront préparées. Ces solutions permettront de vérifier la linéarité.

\section{5.1 Définitions}

Une ligne est un trait continu.

Une relation linéaire en statistique se définit comme une relation où les valeurs varient en restant proportionnelles.

La linéarité d'un test est sa capacité (pour une gamme donnée) à produire des résultats proportionnels à la concentration de l'analyte et à être modélisée par une transformation mathématique.

Le modèle mathématique peut éventuellement être une droite mais s'il est le plus facile à calculer, il s'agit rarement d'un modèle correctement descriptif.

\section{5.2 Types de modélisation}

Si l'on souhaite utiliser une droite comme modèle mathématique, il est indispensable de montrer au moyen d'une analyse de variance type ANOVA que ce modèle est acceptable, c'est à dire que la variation résiduelle n'a pas de contribution significative et que la variation expliquée par la droite décrit bien la variation totale. Une deuxième analyse de variance ANOVA doit s'attacher à montrer que les variations du manquement à l'ajustement n'ont pas de 
contribution significative et que l'erreur analytique pure explique bien la variation résiduelle.

Si l'on ne souhaite pas réaliser de test ANOVA et à chaque fois que cela est possible, nous recommandons et encourageons l'emploi de la régression quadratique.

\section{5.3 Gamme de linéarité}

La gamme de linéarité doit être adaptée en fonction des exigences analytiques. En Toxicologie la gamme dynamique est souvent très étendue puisque l'analyse cherche à couvrir généralement aussi bien les concentrations thérapeutiques que toxiques. L'utilisation de la régression non pondérée (chaque point de la gamme a une importance identique), qui considère que les erreurs en " $y$ " sont identiques pour toutes les valeurs de " $x$ ", ne pourra être valide que si les variances sont homogènes. Nous préconisons l'emploi du test de Hartley ou de Cochran afin de tester les variances (le nombre de mesures pour chaque variance doit être identique pour tous les points de la gamme).

Si les variances ne sont pas homogènes, la régression doit être pondérée par l'affectation d'un poids statistique inversement proportionnel à la variance du point.

Si l'on ne souhaite pas tester l'homogénéité des variances et calculer un modèle pondéré, et à chaque fois que cela est possible, nous recommandons et encourageons l'emploi du calcul mathématique en $1 / x$ ou $1 / x^{2}$, qui représente une approximation très valide de la pondération statistique d'un point de gamme.

Le nombre de points de gamme ne peut être inférieur à 5 et le nombre de mesures par point ne peut être ìnférieur à 3. Dans certains cas particuliers (si l'interprétation ne dépend pas strictement de la concentration), il peut être justifiable de restreindre encore le nombre de mesures.

\section{6 - Stabilité}

Il est nécessaire de définir pcur chaque type d'analyte, en fonction des spécifications techniques du fabricant ou de la connaissance de la molécule de référence, un délai de péremption a priori (1, 3 ou 6 mois...).

A échéance du délai fixé, préparer une nouvelle solution dont les caractéristiques analytiques sont comparées à la solution arrivée à péremption, afin de confirmer ou de modifier le délai de péremption préalablement fixé.

Les critères d'acceptabilité de la validité des solutions de référence sont fixés par le laboratoire pour chaque type d'analyte.

\section{7 - Corrélation de méthodes}

Les concentrations des échantillons de validation doivent être choisies de façon à couvrir l'étendue du domaine d'analyse.

Chacun des $n$ échantillons de validation $(n \geq 5)$, est analysé $p$ fois $(p \geq 3)$ par la méthode A et par la méthode $B$.

1. A partir des valeurs observées, tester, pour chaque méthode, l'homogénéité des variances de chaque échantillon (soit $\mathrm{n}$ variances pour A et pour B) selon par exemple un test de Cochran, de Levene ou de Hartley, etc...(Test d'homoscédasticité)

2. Tester l'égalité des variances entre les deux méthodes selon un test $\mathrm{F}$ à $(\mathrm{p}(\mathrm{n}-1))$ ddl et $(\mathrm{p}(\mathrm{n}-1))$ ddl (Comparaison de deux variances par un test de Fisher).

3. Calculer les différences des résultats obtenus pour chacun des échantillons :

Calculer la moyenne de ces différences ainsi que sa variance et tester l'égalité de cette différence moyenne à zéro selon un test $\mathrm{t}$ de Student, avec $\mathrm{t}$ à (p-1) ddl (Comparaison de moyenne d'échantillons appariés). 


\section{Terminologie - Autres définitions}

La liste des définitions n'est pas exhaustive.

$\underline{\text { Note }}$ : dans la mesure du possible ces définitions ont été reprises à partir des documents qui font références. Dans ce cas l'origine de la définition est indiquée en exposant.

${ }^{1}=$ Vocabulaire international des termes fondamentaux et généraux de métrologie (VIM)

${ }^{2}=$ Guide pour l'expression de l'incertitude de mesure (GUM)

${ }^{3}=$ Statistique : Vocabulaire et symboles NF ISO 3534-1

${ }^{4}$ = Décision de la commission du 7 mai 2002 portant spécifications techniques communes des dispositifs médicaux de diagnostic in vitro [notifiée sous le numéro C(2002) 1344] (Texte présentant de l'intérêt pour l'EEE) (2002/364/CE)

${ }^{s}=$ Norme XPT 90-210 (Protocole d'évaluation d'une méthode alternative d'analyse physico-chimique quantitative par rapport à une méthode de référence) décembre 1999

${ }^{\circ}=$ Guide STP Pharma Pratiques volume $13-n^{\circ} 3$ - mai juin 2003 (Validation des procédures analytiques quantitatives - Harmonisation des démarches)

${ }^{7}=$ Guide de validation des méthodes en biologie médicale doc LAB GTA 04 révision 00-juin 2004

${ }^{8}=$ Techniques de l'Ingénieur, traité Analyse et Caractérisation (Validation interne des méthodes d'analyses)

Acceptabilité, critères d' (Performance standard) ${ }^{7}$ : critères selon lesquels les performances d'une technique sont jugées satisfaisantes dans les conditions d'emploi définies par l'utilisateur (ces critères s'appuient en particulier sur les concepts d'imprécision, d'inexactitude et d'erreur totale acceptable).

Aliquote (Aliquot) ${ }^{7}$ : terme générique se référant à n'importe quelle solution, spécimen etc., dont on utilise uniquement un fragment. C'est une portion mesurée, d'un tout, de composition uniforme.

Analyse, domaine d" : intervalle de concentrations (ou autres quantités) d'un analyte pour lequel la technique est applicable sans modification. Son évaluation nécessite l'établissement des limites de linéarité et (éventuellement) de la limite de détection de la technique. Synonyme : "domaine de mesurage, gamme de mesure, domaine d'application".

Analyte $^{7}$ : composant, substance, matériau à mesurer dans un milieu.

Appareil de mesure ${ }^{1}$ : dispositif destiné à être utilisé pour faire des mesurages, seul ou associé à un ou plusieurs dispositifs annexes.

Biais : voir justesse.

Blanc $^{6}$ : essai réalisé en l'absence de matrice (blanc réactif) ou sur une matrice qui ne contient pas l'analyte (blanc matrice). Par extension, la réponse instrumentale en l'absence d'analyte est utilisée (blanc instrumental).

Bruit de fond ${ }^{7}$ : correspond aux variations aléatoires du signal de mesure pour un niveau donné. Il est mesuré par l'écart-type d'une suite d'au moins 30 mesures du signal, au niveau considéré.

Calibrage $^{1}$ : positionnement matériel de chaque repère (éventuellement de certains repères principaux seulement) d'un instrument de mesure en fonction de la valeur du mesurande.

Note : ne pas confondre « calibrage » et « étalonnage ».

Calibrateur' : matériau de concentration déterminée en analyte soit par pesée, soit par titrage dont le milieu et les propriétés sont imparfaitement connus. Il peut présenter des propriétés physicochimiques nécessitées par une technique.

Coefficient de corrélation ${ }^{3}$ : quotient de la covariance de deux caractères par le produit de leurs écarts-types.

Note : il exprime la relation éventuelle entre deux variables réputées indépendantes. Sa valeur doit être uniquement testée par rapport à zéro en fonction d'un risque $\alpha$ choisi. Il est habituellement sans intérêt dans les comparaisons de techniques.

Coefficient de variation ${ }^{3}$ : pour un caractère non négatif, rapport de l'écart-type à la moyenne.

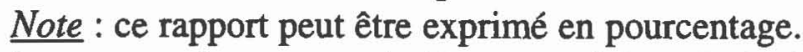

Le terme « écart-type relatif » est parfois utilisé à la place de « coefficient de variation », mais cet usage n'est pas recommandé. 
Composant de type A, (évaluation de l'incertitude) ${ }^{2}$ : méthode d'évaluation de l'incertitude par l'analyse statistique de séries d'observations.

Composant de type B, (évaluation de l'incertitude) ${ }^{2}$ : méthode d'évaluation par des moyens autres que l'analyse statistique de séries d'observations.

Consensus, conférence de $\mathrm{e}^{7}$ : accord exprès ou tacite établi entre les membres d'un groupe institutionnel de concertation, permanent ou temporaire.

Contamination' : phénomène physique lié à un transfert de volume et ayant pour conséquence d'introduire une erreur sur le résultat final.

Contrôle qualité interne (CQI) : il sert à l'évaluation interne des performances du laboratoire en vue de vérifier l'absence de dérive des résultats par mesurage régulier des paramètres contenus dans ces contrôles.

Contrôle qualité externe (CQE) : il sert à l'évaluation des performances du laboratoire par comparaison avec les résultats obtenus par d'autres laboratoires sur le même échantillon.

Corrélation $^{3}$ : liaison entre deux ou plusieurs variables aléatoires à l'intérieur d'une loi.

$\underline{\text { Note }}$ : la plupart des mesures statistiques de corrélation ne mesure que le degré de liaison linéaire.

Cut-off : ce que l'on appelle communément le "cut-off" est la valeur au-delà de laquelle les résultats sont susceptibles de présenter un intérêt biologique ou toxicologique, ou définie par le fabricant.

Degré de liberté $e^{3}$ (ddl) : en général, le nombre de termes de la somme moins le nombre de contraintes sur les termes de la somme.

Ecart $^{1}$ : valeur moins sa valeur de référence.

Ecart-type de la moyenne, s.e.m.("Standard deviation of the mean") ${ }^{7}$ : paramètre statistique indiquant la dispersion des valeurs au niveau de la moyenne d'une série de mesures.

Ecart-type expérimental ${ }^{3}$ : racine carrée positive de la variance.

Ecart-type résiduel, $\mathrm{Syx}^{7}$ : paramètre permettant de chiffrer la dispersion des points autour de la droite de régression.

Echantillon : on appelle souvent "échantillon" la quantité de liquide biologique (ou autre matrice) soumis à l'analyse.

Echantillon de validation : voir standard de validation.

Erreur aléatoire ${ }^{1}$ : résultat d'un mesurage moins la moyenne d'un nombre infini de mesurages du même mesurande, effectués dans des conditions de répétabilité.

Notes:

1. L'erreur aléatoire est égale à l'erreur moins l'erreur systématique.

2. Comme on ne peut faire qu'un nombre fini de mesurages, il est seulement possible de déterminer une estimation de l'erreur aléatoire.

Erreur de justesse (d'un instrument de mesure) ${ }^{1}$ : erreur systématique d'indication d'un instrument de mesure. $\underline{\text { Note }}$ : l'erreur de justesse est normalement estimée en prenant la moyenne de l'erreur d'indication sur un nombre approprié d'observations répétées.

Erreur relative ${ }^{1}$ : rapport de l'erreur de mesure à une valeur vraie du mesurande

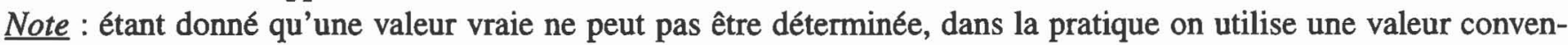
tionnellement vraie.

Erreur systématique ${ }^{1}$ : moyenne qui résulterait d'un nombre infini de mesurages du même mesurande, effectués dans les conditions de répétabilité, moins une valeur vraie du mesurande.

Notes:

1. L'erreur systématique est égale à l'erreur moins l'erreur aléatoire.

2. Comme la valeur vraie, l'erreur systématique et ses causes ne peuvent être connues complètement.

3. Pour un instrument de mesure, voir "erreur de justesse".

Erreur totale ${ }^{7}$ : somme algébrique de l'erreur due à l'inexactitude et de l'erreur due à l'imprécision. Ces deux erreurs se compensent ou s'additionnent, définissant ainsi deux bormes de variation de l'erreur totale. 
Etalonnage ${ }^{1}$ : ensemble des opérations établissant, dans des conditions spécifiées, la relation entre les valeurs de la grandeur indiquée par un appareil de mesure ou un système de mesure, ou les valeurs représentées par une mesure matérialisée ou par un matériau de référence, et les valeurs correspondantes de la grandeur réalisée par des étalons. Notes:

1. Le résultat d'un étalonnage permet soit d'attribuer aux indications les valeurs correspondantes du mesurande, soit de déterminer les corrections à appliquer aux indications.

2. Un étalonnage peut aussi servir à déterminer d'autres propriétés métrologiques telles que les effets de grandeur d'influence.

3. Le résultat d'un étalonnage peut être consigné dans un document appelé certificat d'étalonnage ou rapport d'étalonnage.

Etat de l'art : désigne l'ensemble des méthodes analytiques actuellement disponibles et à utiliser. Il est habituellement estimé à partir d'enquêtes inter laboratoires.

Exactitude $^{6}:$ l'exactitude exprime l'étroitesse de l'accord entre le résultat d'essai et la valeur de référence acceptée, aussi appelé "valeur conventionnellement vraie".

L'étroitesse de l'accord ainsi observée est la résultante de la somme des erreurs systématiques et aléatoires, en d'autres termes l'erreur totale liée au résultat. Par conséquent, l'exactitude est l'expression de la somme de la justesse et de la fidélité.

Evaluation ${ }^{6}$ : étude visant à éprouver le protocole d'analyse (procédure analytique) afin de connaître les valeurs des critères de performance, retenus au préalable. Cette étude peut être menée en intralaboratoire (par le fournisseur ou le fabricant) ou en interlaboratoires.

Fidélité : la fidélité exprime l'étroitesse de l'accord (degré de dispersion, coefficient de variation) entre une série de mesures provenant de multiples prises d'un même échantillon homogène (résultats d'essais indépendants) dans des conditions prescrites.

La fidélité fournit une indication sur les erreurs liées au hasard. La fidélité traduit uniquement la distribution des erreurs aléatoires et n'a aucune relation avec la valeur vraie ou spécifiée.

La fidélité peut être évaluée à trois niveaux :

- la répétabilité : conditions où les résultats d'essais indépendants sont obtenus par la même méthode sur des échantillons d'essais identiques dans le même laboratoire, par le même opérateur, utilisant le même équipement et pendant un court intervalle de temps.

- La fidélité intermédiaire (intralaboratoire) : conditions où les résultats d'essais indépendants sont obtenus par la même méthode sur des échantillons d'essais identiques dans le même laboratoire, avec différents opérateurs en utilisant si possible des équipements différents et pendant un intervalle de temps donné.

- La reproductibilité (interlaboratoires) : conditions où les résultats d'essais indépendants sont obtenus par la même méthode sur des échantillons d'essais identiques dans différents laboratoires, avec différents opérateurs et utilisant des équipements différents.

Grandeur (mesurable) ${ }^{1}$ : attribut d'un phénomène, d'un corps ou d'une substance qui est susceptible d'être distinguée qualitativement et déterminée quantitativement.

Incertitude de mesure ${ }^{7}$ : paramètre, associé au résultat d'un mesurage, qui caractérise la dispersion des valeurs qui pourraient raisonnablement être attribuées au mesurande.

Notes:

1. Le paramètre peut-être, par exemple, un écart-type (ou un multiple de celui-ci) ou la demi-largeur d'un intervalle de niveau de confiance déterminé.

2. L'incertitude de mesure comprend, en général, plusieurs composantes. Certaines peuvent être évaluées à partir de la distribution statistique des résultats de séries de mesurages et peuvent être caractérisées par des écart-types expérimentaux. Les autres composantes, qui peuvent aussi être caractérisées par des écart-types, sont évaluées en admettant des distributions de probabilité, d'après l'expérience acquise ou d'après d'autres informations.

3. Il est entendu que le résultat du mesurage est la meilleure estimation de la valeur du mesurande, et que toutes les composantes de l'incertitude, y compris celles qui proviennent d'effets systématiques, telles que les composantes associées aux corrections et aux étalons de référence, contribuent à la dispersion. 
Justesse (biais) : la justesse exprime l'étroitesse de l'accord entre la valeur moyenne obtenue à partir d'une série de résultats d'essais et une valeur qui est acceptée soit comme une valeur conventionnellement vraie, soit comme une valeur de référence acceptée. La mesure de la justesse est généralement exprimée en terme de biais (erreur systématique). Signalons encore que la justesse était également appelé "exactitude" ou "exactitude de la moyenne".

Limite de détection ${ }^{5}$ : plus petite quantité d'un analyte à examiner dans un échantillon, pouvant être détectée et considérée comme différente de la valeur du blanc (avec une probabilité donnée), mais non nécessairement quantifiée. En fait, il faut prendre en compte deux risques :

- le risque $\alpha$ de considérer la substance présente dans l'échantillon alors que sa grandeur est nulle ;

- le risque $B$ de considérer absente une substance alors que sa grandeur n'est pas nulle.

Limite de quantification ${ }^{5}$ : plus petite grandeur d'un analyte à examiner dans un échantillon pouvant être déterminé quantitativement dans des conditions expérimentales décrites dans la méthode avec une variabilité définie (coefficient de variation déterminé).

Linéarités : capacité d'une méthode d'analyse, à l'intérieur d'un certain intervalle, à fournir une valeur d'information ou des résultats proportionnels à la quantité en analyte à doser dans l'échantillon pour un laboratoire.

Cette proportionnalité s'exprime au travers d'une expression mathématique définie a priori.

Les limites de linéarité sont les limites expérimentales de grandeurs entre lesquelles un modèle d'étalonnage linéaire peut être appliqué avec un niveau de confiance connu (généralement pris égal à $1 \%$ ).

Matériau de référence ${ }^{1}$ : matériau ou substance dont une (ou plusieurs) valeur(s) de la (des) propriété(s) est (sont) suffisamment homogène(s) et bien définie(s) pour permettre de l'utiliser pour l'étalonnage d'un appareil, l'évaluation d'une méthode de mesurage ou l'attribution de valeurs aux matériaux.

Matériau de référence certifié ${ }^{1}$ : matériau de référence, accompagné d'un certificat, dont une (ou plusieurs) valeur(s) de la (des) propriété(s) est (sont) certifiée(s) par une procédure qui établit son raccordement à une réalisation exacte de l'unité dans laquelle les valeurs de propriété sont exprimées et pour laquelle chaque valeur certifiée est accompagnée d'une incertitude à un niveau de confiance indiqué.

Matrice $^{7}$ : milieu dans lequel se trouve l'analyte.

Mesurage $^{1}$ : ensemble d'opérations ayant pour but de déterminer une valeur d'une grandeur.

Mesurande $^{1}$ : grandeur particulière soumise à mesurage.

Méthode : ce terme recouvre l'ensemble des moyens mis en œuvre dans le processus analytique (réactif(s), appareil, mode opératoire, mode de calibrage, mode de contrôle). Le terme de technique n'est pas utilisé en métrologie.

Moyenne, $\mathbf{m}^{7}$ : quotient de la somme des observations par leur nombre. Sauf indication contraire, le terme "moyenne" désigne la valeur arithmétique.

Mode opératoire (de mesure) ${ }^{1}$ : ensemble des opérations, décrites d'une manière spécifique, mises en œuvre lors de l'exécution de mesurages particuliers selon une méthode donnée.

Ordonnée à l'origine (Intercept) ${ }^{7}$ : endroit où la droite d'un graphique coupe l'axe des ordonnées. Désigné conventionnellement par la lettre "a".

Pente (Slope) ${ }^{7}$ : angle que fait une droite sur un graphe par rapport à l'horizontale. Il est exprimé sous forme d'un rapport entre les valeurs de $\mathrm{Y}$ et les valeurs de X. Désigné conventionnellement par la lettre "b".

Performance, critères de $^{7}$ : paramètres caractérisant la procédure analytique (linéarité, fidélité, justesse, ...).

Population ${ }^{7}$ : ensemble (fini ou infini) d'éléments ou d'individus, défini de façons très précises.

Processus de validation d'une méthode d'analyse ${ }^{\mathbf{8}}$ : action de confirmer par examen et apport de preuves tangibles obtenues par des études statistiques intralaboratoires et/ou interlaboratoires que les exigences particulières pour un usage spécifique prévu de la méthode d'analyse sont satisfaites. En général, les exigences particulières portent sur le domaine d'application, la linéarité, la spécificité, la fidélité et la justesse.

Répétabilité : voir fidélité.

Reproductibilité : voir fidélité. 
Résultat d'un mesurage ${ }^{1}$ : valeur attribuée à un mesurande, obtenue par mesurage.

Risque $\alpha$ ou risque de première espèce' ${ }^{7}$ : probabilité de rejeter une hypothèse vraie à l'issue d'un test statistique. Ce risque est fixé avant de réaliser un test (habituellement 5 ou $1 \%$ ). On l'appelle aussi seuil ou niveau d'un test.

Risque $B$ ou risque de deuxième espèce ${ }^{7}$ : probabilité de considérer l'hypothèse nulle Ho comme vraie quand elle est fausse. Le risque $\beta$ dépend du test effectué, du risque $\alpha$ choisi et du nombre de degrés de liberté.

Robustesse : par "robustesse" d'une technique d'analyse, on entend une mesure de sa capacité à ne pas être affectée par des variations faibles mais délibérées des paramètres de la méthode, et qui fournit une indication sur sa fiabilité dans les conditions normales d'utilisation.

Sensibilité : une méthode est dite "sensible" si une faible variation de la concentration ou de la quantité d'analyte entraîne une variation significative de la réponse.

Il convient d'éviter de définir, par extension, la sensibilité de la méthode comme sa capacité à détecter de faibles concentrations.

Série $^{7}$ : ensemble de mesures consécutives effectuées sans interruption et dont les résultats sont obtenus à partir d'une phase unique de calibrage.

Signal de mesure ${ }^{1}$ : grandeur qui représente le mesurande et qui lui est fonctionnellement liée.

Spécificité : la spécificité d'une procédure analytique est sa capacité à établir de manière univoque l'existence d'une substance à analyser en présence d'autres composants potentiellement présents.

Standard d'étalonnage : voir matériau de référence.

Standard de validation ${ }^{6}$ : les standards de validation sont des échantillons, reconstitués dans la matrice ou tout autre matériau de référence, dont la valeur vraie a pu être établie par consensus et qui sont utilisés pour la validation de la procédure d'analyse.

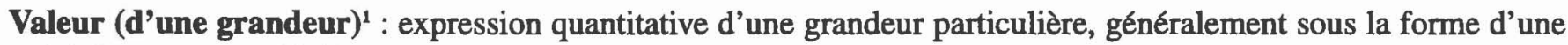
unité de mesure multipliée par un nombre.

Valeur conventionnellement vraie (d'une grandeur) ${ }^{1}$ : valeur attribuée à une grandeur particulière et reconnue, parfois par convention, comme la représentant pour une incertitude appropriée pour un usage donné.

Validation (validation des méthodes) : cf. définition de l'ISO/CEI 17025, au chapitre I Introduction.

Variance $^{7}$ : paramètre statistique chiffrant la dispersion des individus autour de la moyenne. C'est la somme des carrés des écarts à la moyenne de $\mathrm{n}$ observations divisée par le nombre de degrés de liberté. L'écart-type est la racine carrée de la variance.

Vérification : confirmation par examen et établissement des preuves que les exigences spécifiées ont été satisfaites.

\section{Bibliographie}

- Analyse des produits agricoles et alimentaires - Procédure de validation intralaboratoire d'une méthode alternative par rapport à une méthode de référence - Cas de méthodes d'analyses quantitatives. Norme NF V03-110, décembre 1998.

- Vassault A., Grafmeyer D., de Graeve J., Cohen R., Beaudonnet A., Bienvenu J. Analyses de biologie médicale : spécification et normes d'acceptabilité à l'usage de la validation des techniques. Ann. Biol. Clin. 1999 ; 57(6) : 685-95.

- Application de la statistique - Exactitude (justesse et fidélité) des résultats et méthodes de mesure, parties 1 à 6. Norme NF ISO 5725, décembre1994 et octobre 1998.

- Journal officiel de la République française. Arrêté du 26 novembre 1999 relatif à la bonne exécution des analyses de biologie médicale. (GBEA). 11 décembre $1999 ; 287: 18441$.

- Clinical Laboratory Improvement Amendments (CLIA). http://www.cms.hhs.gov/clia (site consulté en mai 2005). 
- Désenfant M., Priel M., Rivier C. De la validation des méthodes d'analyse à l'évaluation de l'incertitude des résultants de mesure. Actes du $11^{\text {eme }}$ congrès international de métrologie. Toulon, 20-23 octobre 2003. Document téléchargeable sur le site : http://www.Ine.fr/publications/actes_11e_congres_metrologie/81.pdf

- Comité Français d'Accréditation (COFRAC). Document 1133. Procédure de validation interne des méthodes d'essais. Octobre 1995 ; document téléchargeable sur le site : http://www.cofrac.fr/doc/docs/1_laboratoires/essais/1133v00.doc

- Jansen R.T.P. Essential criteria for quality systems of medical laboratories. Eur.J. Clin. Chem. Clin. Biochem. $1997 ; 35(2): 123-32$.

- Perruchet C., Priel M. Estimer l'incertitude, in : Mesures - Essais., Paris, Edition AFNOR, 2000.

- Duval C. Evaluation de l'automate Axsym®. Ann. Biol. Clin. 2001 ; 59 (5) : 48-51.

- Évaluation des performances des dispositifs médicaux de diagnostic in vitro. Norme NF EN 13612, septembre 2002.

- Warnet J.M. Evaluation d'une technique de détermination de l'éthanolémie par chromatographie en phase gazeuse. Ann. Biol. Clin. 1990 ; 48(8) : 587-95.

- Guide de validation analytique. SFSTP. STP Pharma. Prat. 1992 ; 2 : 205-26.

- Guide de validation des méthodes de dosage biologique. SFSTP. STP Pharma. Prat. 2002 ; 12 (6) : 317-36.

- Quantifier l'incertitude dans les mesures analytiques. Guide EURACHEM/CITAC, $2^{\text {eme }}$ éd., 2000 ; document téléchargeable sur le site : http://www.lne.fr/fr/services_ligne/guides_documents techniques.shtml

- Rivier C., Lalere B. Guide méthodologique pour l'estimation des incertitudes en analyse chimique. Actes du $11^{\text {ème }}$ congrès international de métrologie. Toulon, 20-23 octobre 2003. Document téléchargeable sur le site :

http://www.lne.fr/fr/service_ligne/publications.shtml

- Guide pour l'expression de l'incertitude de mesure. Paris, ISO, 1995.

- Feinberg M., La validation des méthodes d'analyse. Paris, Masson, 1996.

- Laboratoires d'analyses de biologie médicale - Exigences particulières concernant la qualité et la compétence. Norme NF EN ISO 15189, octobre 2003.

- Groupe de travail SFBC. Le protocole Valtec : évolution du concept et du contenu. Ann. Biol. Clin $1997 ; 55(2)$ : 167-73.

- Groupe de travail SFBC. Le protocole Valtec : protocole de validation de techniques (document B). Ann. Biol. Clin $1986 ; 44$ 686-745.

- Guide EA-4/16. Lignes directrices d'EA pour l'expression de l'incertitude des résultats d'essais quantitatifs. 2004. Document téléchargeable sur le site : http://www.lne.fr/publications/guides-documents techniques .shtml

- Lignes directrices relatives à l'utilisation d'estimations de la répétabilité, de la reproductibilité et de la justesse dans l'évaluation de l'incertitude de mesure. Norme ISO/TS 21748, 2004.

- Widmer-Girod C., Staub C. L'incertitude de mesure : une notion à définir en toxicologie médico-légale. Ann. Toxicol. Anal. $2004 ; 16(3): 215-9$.

- Manuel suisse des denrées alimentaires. Analyse des produits agricoles et alimentaires. Guide pour la validation des méthodes d'essai physico-chimiques et évaluation de l'incertitude de mesure. Chapitre 60C, 1998.

- Méthodes chromatographiques de dosage dans les milieux biologiques : stratégie de validation : SFSTP. STP Pharma. Prat. 1997 ; 7 : 169-94.

- Normes fondamentales - Vocabulaire international des termes fondamentaux et généraux de métrologie. Norme NF X 07-001, décembre 1994.

- Prescriptions générales concernant la compétence des laboratoires d'étalonnages et d'essais. Norme NF EN ISO/CEI 17025, mai 2000.

- Procédure de validation d'une technique. Spectra Biol. 1997 ; 16(90) : 43-50. 
- Programmes d'accréditation COFRAC de Biologie Médicale : Programme n 143 Analyses en Biochimie. Révision 03, mai 2003. Document téléchargeable sur le site : http://www.cofrac.fr/doc/docs/1_laboratoires/essais/p143.doc ; Programme 168 Analyses en Toxicologie et Suivi Thérapeutique Pharmacologique. Version 00, mai 2002. Document téléchargeable sur le site http://www.cofrac.fr/doc/docs/1_laboratoires/essais/p168v0.doc.

- Qualité de l'eau - Protocole d'évaluation d'une méthode alternative d'analyse physico-chimique quantitative par rapport à une méthode de référence. XP T 90-210, décembre 1999.

- Nicolas O., Farenc C., Bressolle F. Stratégie de validation de méthodes de dosage en bioanalyse en vue d'études pharmacocinétiques et toxicologiques. Ann. Toxicol. Anal. $2004 ; 16(2)$ : 118-27.

- Feinberg M. Validation interne des méthodes d'analyse. Techniques de l'Ingénieur, pp 224-1 - 224-23.

- Validation des procédures analytiques quantitatives - Harmonisation des démarches. SFSTP. STP Pharma. Prat. $2003 ; 13(3)$ : 101-38.

- Clerc Y., Huart B., Charotte J,M, Pailler F.M., Validation d'une méthode de dosage de l'éthanol sanguin par chromatographie en phase gazeuse. Ann. Biol. Clin. 1995 ; 53(4) : 233-8.

- ICH harmonised tripartite guideline. Validation of analytical procedures Q2A., International conference on harmonisation of technical requirements for registration of pharmaceuticals for human use ; 27 octobre 1994.

- ICH harmonised tripartite guideline. Validation of analytical procedures : methodology Q2B. International conference on harmonisation of technical requirements for registration of pharmaceuticals for human use; 6 novembre 1996. 